\title{
Practical Guidelines for Studying Young Children With Autism Spectrum Disorder in Psychophysiological Experiments
}

\author{
Anneli Kylliäinen • Emily J. H. Jones • Marie Gomot • \\ Petra Warreyn • Terje Falck-Ytter
}

Received: 27 August 2014 / Accepted: 2 September 2014 / Published online: 12 September 2014

(C) The Author(s) 2014. This article is published with open access at Springerlink.com

\begin{abstract}
Understanding neurocognitive mechanisms in children with autism spectrum disorder (ASD) is an essential goal of autism research. Studying young children with ASD or other neurodevelopmental conditions in demanding experimental settings, however, can pose many practical and ethical challenges. In this article, we present practical strategies that facilitate data acquisition from psychophysiological experiments involving young children with ASD. We focus on a range of common, non-invasive technologies including EEG, MEG, eye tracking as well as some common measures of physiological arousal. Topics have been divided according
\end{abstract}

A. Kylliäinen $(\triangle)$

Human Information Processing Laboratory, University of Tampere,

33014 Tampere, Finland

e-mail: anneli.kylliainen@uta.fi

\section{A. Kylliäinen}

Autism and Development Team, Institute of Psychiatry, King's

Collage London, London, UK

E. J. H. Jones

Centre for Brain and Cognitive Development, Birkbeck College,

London, UK

M. Gomot

UMR 930, INSERM, Université François-Rabelais de Tours, Tours, France

P. Warreyn

Department of Experimental Clinical and Health Psychology, Ghent University, Ghent, Belgium

T. Falck-Ytter

Center of Neurodevelopmental Disorders at Karolinska Institutet

(KIND), Stockholm, Sweden

T. Falck-Ytter

Uppsala Child \& Babylab, Department of Psychology, Uppsala

University, Uppsala, Sweden to the chronological order of the experimental procedure: (a) design, (b) preparing for the measurement visit, (c) conducting the experiment and (d) the data handling. A key theme in the proposed guidelines is the difficulty in balancing the procedural adaptations necessary to facilitate participation of children with ASD, and maintaining standardisation for all participating children.

Keywords Autism spectrum disorder.

Psychophysiological measures - EEG - Eye tracking · Guidelines

\section{Introduction}

Recent research suggests that autism spectrum disorder (ASD) is a neurodevelopmental disorder caused by a complex interaction between a range of genetic and environmental factors (e.g. Sandin et al. 2014). However, the precise pathways by which these factors lead to the behavioural symptoms of ASD remain largely unclear (Hallmayer et al. 2011). ASD is currently diagnosed on a purely behavioural basis and is defined by a set of specific symptoms including qualitative impairments in social communication and the presence of restricted and stereotyped behavioural patterns (American Psychiatric Association 2013). Characterising causal pathways to ASD, and finding ASD specific biomarkers, requires moving beyond the behavioural level to study the neurocognitive systems that underpin the atypical development. So far, these neurocognitive functions - which may underlie the abnormal development in ASD - have been studied most intensively in high-functioning school-aged children and adults with ASD, limiting the scope of current knowledge. In addition to the 'pure' effects of age, high functioning individuals with ASD may use unique compensatory strategies that further blur our understanding of the core underlying mechanisms. Against 
this background, it is clear that studying neurocognitive mechanisms in young children with ASD is an important goal for the field (cf., Dyckens and Lense 2011; Itier and Batty 2009). Moreover, due to technological developments, such studies are increasingly feasible (e.g. Holmqvist et al. 2011; LloydFox et al. 2010).

Research with neuroimaging techniques, including electroencephalography (EEG), magnetoencephalography (MEG), functional magnetic resonance imaging (fMRI) and Near Infrared Spectroscopy (NIRS), has revealed a range of atypicalities in neurocognitive systems in toddlers and young children with ASD. For example, the excellent temporal resolution of EEG and MEG has revealed abnormalities in basic auditory (e.g. Gomot et al. 2011; Orekhova et al. 2008) and visual (e.g. Cléry et al. 2013) discrimination, in addition to atypicalities in social processing (e.g. Grice et al. 2005; Kylliäinen et al. 2006; Senju et al. 2005; Webb et al. 2011). EEG has also proved sensitive to the effects of early intervention, indicating its utility as measure in clinical trials (Dawson et al. 2012). Also traditional psychophysiological measures such as skin conductance responses (SCRs) and measures derived from heart rate have indicated differences in modulation of arousal, which may be related to atypical social, emotional and attentional processing in autism (e.g. Hirstein et al. 2001; Joseph et al. 2008; Kylliäinen and Hietanen 2006; Kylliäinen et al. 2012; Patriquin et al. 2013; Sigman et al. 2003; Watson et al. 2012). Finally, tracking of eye movement using infrared cameras has revealed a range of differences in the way visual attention is deployed in young children with autism (e.g. Chawarska and Shic 2009; Falck-Ytter et al. 2013a, b, c; Jones et al. 2008; Klin et al. 2009; Swanson and Siller 2013).

Whilst neuroimaging and psychophysiological techniques have become increasingly child-friendly over time, the unusual sensory behaviours and difficulties in social communication and restricted and stereotyped behavioural patterns of ASD can pose significant challenges to investigators using these techniques. When studying young, newly diagnosed children with ASD, they often also experience developmental delay that necessitates the inclusion of a control group of children that have developmental delay without marked autistic behaviour to the study design. It can be difficult for children with ASD or other neurodevelopmental conditions to comply with common experimental paradigms designed for typically developing children, which can lead to a significant attrition rate. For example, neuroimaging methods are usually sensitive to many sources of artifact, such as movements of the child, which can reduce the quality of the data. Further, it is critical to avoid making modifications to the experimental setup that create systematic differences in data collection methods between the children with additional needs and typically developing children, thus causing significant confounds in experimental data.

The aim of this article is to introduce practical strategies for optimising data acquisition in neuroimaging and psychophysiological experiments involving young children with ASD and other developmental disorders. Due to our focus on issues in children with an ASD diagnosis, we do not deal with issues related to testing infants (e.g. infants at high familial risk for ASD) since many of the practical challenges associated with ASD relate to behavioural symptoms or associated comorbidities that do not emerge until toddlerhood or later (Jones et al. 2014). Moreover, instead of focusing on one specific method and the accompanying technical issues (cf., Webb et al. 2013), we will be covering practical strategies that are applicable across the range of common techniques that are used in psychophysiological studies in young children with ASD (EEG, MEG, eye tracking and measurements of heart rate and skin conductance). Further, we have a particular focus on the practical aspects of studying young children with ASD and other neurodevelopmental conditions. Experienced researchers typically follow explicit and implicit principles when designing their studies and employ a range of support strategies in order to help children with special needs participate successfully in these experiments. However, these principles and strategies are often not fully documented in empirical reports, in part due to space constraints. Furthermore, most of the strategies are feasible when studying school-aged children with ASD but need modifications when moving towards younger and more low-functioning children. In this article, we will concentrate on the practical guidelines for supporting young and possibly low-functioning children with ASD. Although many of these guidelines will apply also to other groups of children with other neurodevelopmental disorders, some of the aspects are ASD specific. Additionally, we will raise some of the issues that need to be considered when planning the design and analysing the findings of the studies of ASD and other neurodevelopmental conditions.

\section{Practical Guidelines}

The practical strategies and other topics to be considered, when studying young children with ASD in psychophysiological studies, have been divided according to the chronological order of the experimental procedure. The phases of (a) design, (b) preparing for the measurement visit, (c) conducting the experiment and (d) the data handling will be discussed separately although these phases are partly overlapping. The specific topics that should be taken into account in different phases of the experiment are summarised in Table 1.

\section{Design}

\section{Stimuli Selection}

Selection of stimuli must reflect not only theoretical questions, but also practical considerations. When using auditory or 
Table 1 The topics to consider when studying young children with ASD in psychophysiological experimental settings

\begin{tabular}{|c|c|c|c|}
\hline Design & Preparing for the measurement visit & Conducting the experiment & Data handling \\
\hline $\begin{array}{l}\text { Stimuli selection } \\
\text { Experiment duration } \\
\text { Attention grabbers } \\
\text { Active vs. passive task }\end{array}$ & $\begin{array}{l}\text { Preparation of the testing environment } \\
\text { Using parental knowledge } \\
\text { Desensitising }\end{array}$ & $\begin{array}{l}\text { Warm up and preparation } \\
\text { Physical support } \\
\text { Behavioural control strategies } \\
\text { Experimenter characteristics } \\
\text { Difficult situations }\end{array}$ & $\begin{array}{l}\text { Feedback to the child and the parents } \\
\text { Online strategies for recording behaviour } \\
\text { and other factors } \\
\text { Offline strategies to reduce data loss }\end{array}$ \\
\hline
\end{tabular}

visual stimuli, it is good to be aware that children with ASD might be over- or under-sensitive to some visual and auditory stimuli (e.g. Marco et al. 2011; Stiegler and Davis 2010). Children with ASD indeed often exhibit atypical sensory behaviours such as auditory self stimulation or conversely noisy environment avoidance that suggest unusual sensory processing. Systematic review of the literature indicates that rates of sensory processing dysfunction may be as high as $90 \%$ in individuals with ASD (Baker et al. 2008; Blakemore et al. 2006; Leekam et al. 2007; Tomchek and Dunn 2007), and indeed, sensory sensitivities are now included in DSM-5 criteria for ASD (American Psychiatric Association 2013). Abnormal responses to stimuli seem to affect all sensory modalities and not only include enhanced perceptual function such as visual hyperacuity (Ashwin et al. 2009), hyperacusis (Khalfa et al. 2004) and acute tactile sensitivity (Blakemore et al. 2006), but also hyporeactivity to sensory stimuli which has been extensively reported in all sensory modes (BenSasson et al. 2009; Reynolds and Lane 2008). The emergence of some autistic behaviour may stem from these sensory impairments (see for comprehensive review, Gerrard and Rugg 2009) and might be unintentionally triggered by stimuli used in the experiments. In order to avoid such extreme reactions to stimuli, sounds higher than $70 \mathrm{~dB}$ SPL should be avoided. The auditory stimuli could be delivered by speakers if headphones are not tolerated. If possible, fast moving visual stimuli and flickering light should be avoided keeping in mind visual oversensitivity (Stiegler and Davis 2010) and difficulties of children with ASD in movement and speed processing (Gepner and Féron 2009) when these processes are not the target of the study. Tactile sensitivity may also impact inclusion rates when using recording methods that involve skin contact (cf., Webb et al. 2011). In addition to taking into account these general guidelines, researchers should always pilot their stimuli in a small sample of children with ASD to make sure that there are no unexpected reactions to the stimuli.

Video presentation is often preferred over live presentation because it is easier to control in experimental settings (cf., Falck-Ytter et al. 2013a, b, c). Dependent on the content, computerised stimuli may elicit increased attention and reduce motor behaviour in children relative to live stimuli. In addition, it offers a standardized presentation of the stimuli (Barr et al. 2007) and facilitates synchronisation with the EEG data. However, the processing of 2D (video) versus 3D (live) presentation may be different in young children (cf., Carver et al. 2006). It has indeed been found that typically developing infants show a reduced brain response to video compared to live presentation (Ruysschaert et al. 2013; Shimada and Hiraki 2006). Therefore, the choice for video presentation should be supported by literature or pilot data showing that the perception and processing is similar to that of live presentation, also in the children with ASD. It should be noted, however, that in some circumstances children with ASD might find the presence of a live interacting person more stressful than seeing the person through the monitor. In case the life vs. video presentation is not the target of the study, the pilot testing should help to decide which stimulus presentation mode to use. During the pilot testing, it is also good to control the level of attentiveness to the movie in every participating group.

\section{Experiment Duration}

Short attention span, lack of motivation to comply other's demands and communication difficulties can constrain experiment length in special populations such as young children with ASD. First of all, the duration of the measurement time cannot be very long especially when most of the measurement methods demand that the child is ought to stay rather still. Piloting the experiment with the child with special needs is important in order to find the optimal duration of the certain experiment. Depending the experimental setting and used support strategies, a high-functioning 7 years of age child with ASD could tolerate up to 20-30 min of measuring time (cf., Kylliäinen et al. 2006; Kylliäinen et al. 2012); whereas with a low-functioning 3-year-old child, uninterrupted measuring time could, for example, vary from 2 to $10 \mathrm{~min}$ only. In the study of Ruysschaert et al. (2014), 2- to 5-year-old children with ASD were able to conduct a 20-min EEG experiment, but they were allowed to move in between presentations.

To increase measuring time by supporting the child's vigilance and motivation to carry on previous eye tracking and EEG -studies have utilized methods in which the stimuli in different sub-studies are either mixed together (e.g. FalckYtter et al. 2012) or alternatively presented in changing blocks (Falck-Ytter 2010; Warreyn et al. 2013). It could be, however, 
that some effects do not replicate when stimuli are mixed instead of presenting them in the separate blocks or studies. Further, the possibility of differential habituation rates between groups should be considered. Evidence suggests that ERP responses habituate over time in developing populations (Snyder et al. 2002), and behavioural evidence suggests that children with ASD show slower habituation to faces than children with typical development (Webb et al. 2010).

In paradigms requiring multiple trials, researchers must thus determine whether to present stimuli until children stop paying attention or whether to present a set number of stimuli to all children. Choosing the former option can result in a greater number of valid trials for analysis and ensures that processing is sampled across the same range of behavioural states for each group (from attentive to inattentive). However, this is likely to result in many more valid trials for the control group of typically developing children than the groups of children with ASD and children with developmental delay without ASD.

Another strategy to increase the quality and the amount of data is to have a break with the child. If acceptable with the parents, the child could be offered something to eat or drink during the break as a reward and refreshment. However, it is important not to increase the length of the break so that the child would have attention resources left to carrying on in the experiment. Further, it is important to match breaks across groups, since changes in state induced by breaks could significantly impact the results of some studies.

\section{Attention Grabbers}

Sometimes it might be difficult to carry on after the break and more useful method to increase the child's motivation and vigilance could be the use of 'break videos' ('attention grabbers') in between trials. Typically, the attention grabber involves auditory or visual signals specifically designed to direct the child's attention to the desired location. When studying young children, the use of attention grabbers is crucial and serves many functions. Visual signals are typically used at the beginning of the trial in order to get child's attention towards the screen and towards a specific fixation point on the screen. This kind of attention grabber could be for example a slowly flashing, colourful circle (e.g. Peltola et al. 2008) or a cartoon image in the middle of the screen (e.g. Senju and Csibra 2008). Some auditory cues, such as the sound of a bell, can be used to simply disrupt the child's current activity, alert him/her and result in the child looking back towards the stimulus or the auditory signal could be accompanied with the visual attention grabber at the beginning of each trial.

Attention grabber movies, shown in-between the actual stimuli, can be used in computer based experiments and these animations are designed for the sole purpose of getting the child's attention and motivate him/her to carry on in the experiment. Even though it is recommended to use child friendly stimuli, one should be cautious in using very popular cartoon characteristics in case some of the children have specific preoccupations to them. To avoid this kind of situation, it is good to have an alternative set of attention grabbers and make sure from the parents beforehand what type of attention grabbers are the most optimal to use. It is also good to avoid too arousing break videos and present them only for few seconds not to unnecessarily increase the length of the experiment.

The transition from one stimulus to the next or from an attention grabber to the next stimulus can either be fixed or dependent on the child's behaviour. The latter can involve manually controlled transitions in which the experimenter is observing the child and determines when to continue the presentation. When an eye tracking method is used, automatic gaze contingent techniques can be utilised, in which the transition between the attention grabber and the stimulus is based on eye movement data from the child and the computer software decides when to continue. These can also be used to ensure that stimuli are presented if and only if the child is attentive (looking), although the efficiency of such techniques depends on the ability of the equipment/software to correctly classify fixations online. Generally speaking, building in the option of flexible transitions can dramatically increase the data from any experiment with young children. Controlling the transitions enables the experimenter to make short adjustments ('Look at the screen!') as well as somewhat longer ad hoc breaks.

\section{Active vs. Passive Task}

One further issue to consider, when planning the design for young children with ASD, is whether the use of any kind of active task would increase the concentration and motivation during the psychophysiological measurement. Nevertheless, the cognitive challenge of the task needs to be carefully considered as young children with ASD might not understand the verbal instructions and could be functioning at a low developmental level. It can be advantageous if the rule is visually obvious so that no verbal instructions are needed. For example, in Kylliäinen et al.'s on-going EEG-study, 3 to 5 years of age, low-functioning children with ASD are asked to press either a red or a green button when they see a photo image of either of the buttons on the screen. The task is irrelevant to the study question, and it is there only for supporting the attention and motivation of the child. In contrast, for the young and low-functioning children, it may be equally good to use only a passive task, e.g. only sit and watch a movie whilst for example the auditory EEG-responses are being measured (cf., Gomot et al. 2011). 
The trouble to avoid in the active tasks is that the child might be tempted to press the button repeatedly and also during the measurement times. This could be avoided by giving the buttons for handling only when there is an interval for the task or by covering the button with the experimenter's hand when it is not the time for pressing it. If an active 'supporting' task is introduced, it is important to consider whether group differences in task performance may cause unwanted effects on the variables investigated in the study.

Preparing for the Measurement Visit

\section{Preparation of the Testing Environment}

When studying young children in experimental setups, the imaginative scenery of the testing room is sometimes used. The laboratory can be designed, e.g. as a spaceship (Schoen et al. 2008) or a scene of a familiar cartoon. Having playful and imaginative settings might help the majority of children without ASD. It is not known, however, how these kind of contextual factors influence young children with ASD given their difficulties in imagination. It might be a confounding factor when interpreting the possible group differences if one group of children imagined themselves being spacemen and another group of children could not imagine themselves being anything else than they are. Furthermore, a lot of stimulation could also increase the arousal level of the children too much which might in turn affect measurements and make concentration hard. It is a risk, in any case, that the context modulates performance differently in children with and without ASD.

The preferred first choice would probably be to keep the testing environment as simple as possible, keeping also in mind the possible sensory hypo- or hyperreactivity of the children with ASD. It would also make it easier the use of stimulus control strategies, i.e. control behaviour through increasing the saliency of stimuli that are discriminative for the desired behaviour, and removing stimuli that are discriminative for other, unwanted behaviour. For example, all the distracting materials should be removed from the testing room and an attractive toy could be placed on the chair in which the child is ought to sit or an interesting children's movie could be on-going on the screen when the child is entering the testing room. It is also important to be able to adjust the lightening in the room and keep this constant between the participating groups. This is particularly essential when using eye tracking, in which the quality of data may change when the lightening conditions change.

\section{Using Parental Knowledge}

The help of the parents is crucial when studying children with special needs and especially children with autism who usually have unusual preoccupations and behaviours. The parents are a good source of information prior the laboratory visit. It is highly advisable to talk with the parents beforehand. It can be very useful to go through the experimental setting in detail with the parents and ask them which part of the procedure might most likely cause some difficulties with their child (cf., Kylliäinen et al. 2006, 2012; Falck-Ytter et al. 2013a, b, c). When explaining the procedure, one can ask whether the parents think it is realistic to comply with their child, what they think can be done to help the child and if there is anything that should be avoided. This kind of careful preparation may help avoiding later problems and unrealistic expectations on the child. In studies that the child have to wear an EEG cap/net or leads for recording, e.g. SCR, heart rate and oculomotor activity, the parents could give information about their child's tolerance of them. Sometimes the parents are, however, amazed how well their child could tolerate the measuring demands although they anticipated difficulties. It is essential to inquire whether the child is afraid of anything and whether there are specific things to avoid. Trying to avoid things that might upset the child is not only important ethically, but children with ASD might be more difficult than typically developing children to calm down and make settle if something goes wrong and upset them, e.g. recycling bins in a corridor that leads to a test room. Another source of relevant information could be the knowledge from the clinicians and the teachers who have worked with the child. They may have very detailed information about the realistic expectations of the particular child, and how they usually support him/her.

\section{Desensitising}

When the experimental method requires the child to wear a cap, a helmet or a net and leads attached to the child's skin, the additional desensitising visit before the actual measuring is recommended. Sometimes young children with ASD have not seemed to be helped by extensive desensitisation right before the measurement session and a separated desensitising visit has shown to be preferable (cf., Webb et al. 2013). During the desensitising visit, the child can try an EEG cap or sit under a mock scanner. For example, in a magnetoencephalography study by Kylliäinen et al. (2006), a mock scanner was created. The participating school-aged children were invited for the prior desensitising visit.

The young children could also benefit practicing the measurement demands by taking a practice teddy who wears leads to home with. The parents could also be given practice stickers. One should be aware though that too intensive practicing could also lead to a situation that the child learns to resist using stickers and leads. Therefore, the practising with the stickers is recommended to do without actually attaching the sticker to the child's skin. One option could be to show to the child and the parents a preparation video in which someone else is going through the measurement. The visit before 
the actual experiment gives also an opportunity for the experimenter to meet the child and become aware of the individual needs and to talk with the parents. The pre-visits are also quite often used for the clinical assessments, such as ADOS assessment (Lord et al. 2012) and assessment of cognitive abilities or developmental level.

\section{Conducting the Experiment}

\section{Warm up and Preparation}

The purpose of the warm up phase is to reduce arousal and anxiety associated with the general lab environment and the experimenter(s), whereas the purpose of the desensitising is to reduce arousal and anxiety associated with specific stimuli or equipment. The preparation refers to measurement preparations which are needed in particular psychophysiological measures, such as attaching the electrodes on a child, measuring the head position in the helmet and adjusting the impedances to the best possible level. Although the aim is to keep the experimental procedure relatively similar for all the participating children, the individual modifications in pacing the warm up and preparation period are usually needed. Nevertheless, the procedure as a whole should not be very different for the children in the control groups although they might not have similar needs than children with ASD.

On arrival to a research laboratory, several studies (e.g. Kylliäinen et al. 2006, 2012; Ruysschaert et al. 2014; Warreyn et al. 2013) have adopted a strategy that there are two experimenters welcoming the family. One experimenter is taking care of the parents by explaining the procedure and the parental role and the second experimenter can concentrate on the child only.

The time used for warming up depends on the child's developmental level and personality together with the used method. For example, some of the eye tracking studies including young and low functioning children with ASD has found it beneficial to start quickly after the entering the testing room (Falck-Ytter et al. 2013a, b, c). Starting quickly can reduce the risk that the child gets restless as unstructured situations with social demands are usually very stressful for the children with ASD. The experimenter is often new to the child, and this 'novelty effect' may make the child more attentive and responsive to clear prompts and instructions, particularly directly after arrival. Getting the child to start the experiment quickly also guarantees that the attention resources of the child are being used in the best possible way. Of course, a shy child might require a bit longer warm up period.

Methods that require more preparation (e.g. EEG, MEG) before the actual measurement could need more warm up time and clarification of the procedure especially if there has not been a desensitising visit beforehand. It is recommended to start preparation by making sure that the child has visited a toilet recently, especially with the child that does not use nappies anymore. In case a pre-visit is not feasible, a sufficiently long warm-up or desensitising period should be planned before starting the actual experiment. In an EEG study examining mu suppression in young children with ASD (Ruysschaert et al. 2014), care was taken never to plan a second appointment within $4 \mathrm{~h}$ after the booking for a child with ASD, although the actual experiment took less than $2 \mathrm{~h}$.

The use of children's cartoons and videos have been utilised during the EEG preparations (Ruysschaert et al. 2014; Warreyn et al. 2013). The use of child's own favourite videos might be considered, but it should be kept in mind that a young child with ASD might have preoccupations to his/her own videos and might get upset when it is time to stop watching it and start the experiment. Thus, it could be more optimal to use set of same videos for all the children and check from the parents that the preparation video is not the one that might cause problems. For young children directing their attention away from EEG-net/cap preparation or lead preparation is a one good strategy, the older children with ASD might need to know exactly where the leads would be attached and they might need rewards after each lead (cf., Kylliäinen et al. 2006). The experimenters have to make sure that the attaching the leads and preparing the helmet or net will not take too long. Again, if the child tolerates the close presence of two persons, it may be useful to have two experimenters available, e.g. for inserting the electrolytic gel into EEG electrodes. In some studies, a group of typically developing children have been measured first in order to get enough practice of the preparation and of the whole procedure (e.g. Kylliäinen et al. 2006; Ruysschaert et al. 2014).

\section{Physical Support}

The psychophysiological measures are all quite sensitive to the movements of the child. A chair that is adjustable and supports the sitting position from neck to feet is therefore essential and not only to help the child to sit still, but also to structure the testing environment for the child and support his/ her attention. The height of the chair needs to be easily adjustable and if the chair has wheels they need to be possible to lock. In case lateral stimuli are used, it is important that the chair supports head position. Young children are not usually agreeable to use a chin rest. There could be belts in the chair to support the child's posture and preventing the child to get out of the chair too quickly. It should be noted, however, that not all the children tolerate the use of belts. The car seat for children is quite often used and in this context the children are used to wear seatbelts. Seats that are meant for the children with physical disabilities are found to be a good choice (Fig. 1).

Depending on the child's age, sitting on the parent's lap is sometimes an option. In that case, it should be kept in mind 


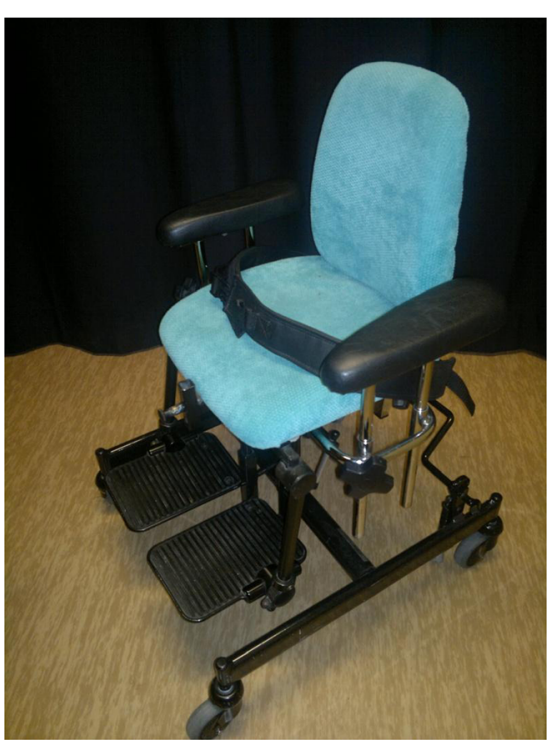

Fig. 1 An example of a chair that can be used for physical support during the measurement

that some children with ASD do not find it helpful and typically developing young children usually do more social referencing with their parents during the experiment than children with ASD. The social referencing causes not only artefacts but might affect findings of the group differences. Indeed, it has been found that joint attention with a parent can influence ERP components (Carver et al. 2006), and the ability to respond to those cues may differ between groups. Also when measuring physiological arousal, it might be difficult to control touching when the child is sitting on the parent's lap. Nevertheless, in some EEG-studies (e.g. Ruysschaert et al. 2014; Warreyn et al. 2013), it has been found preferable that the participating toddlers sit on their parents lap whilst measuring. In those studies, parental interference has been minimised by explicitly instructing the parents to remain silent, respond minimally their child's initiations and simply ask their child to carry on attending the stimuli. Sitting on the parents lap has been found to reduce movements, and the parents can also often prevent (or stop) the children from touching or pulling the cap or the electrodes. Which seating arrangement is the most optimal for the certain experiment depends on the child's developmental level and the study requirements. The seating arrangements should be, however, kept as similar as possible for all the children in different groups.

\section{Behavioural Control Strategies}

Planned and systematic use of behavioural control strategies, such as rewarding and positive reinforcement is a crucial support for the children with ASD, and it is usually beneficial for all the other children too. Rewarding typically refers to object or events that we believe should be experienced as positive by the child. Positive reinforcement refers to an increase in a response due to contingent delivery of (reinforcing) stimuli. Positive reinforcement is one of the main principles of behaviour change according to (operant) learning theory. The main aim of behavioural control strategies is to increase the probability that the child will be attentive and to tolerate the actions that he/she does not necessarily find inherently rewarding (like sitting on a chair for several minutes). In one single experimental trial, it may not always be possible to achieve behavioural change through operant conditioning, and thus the primary control strategy one has access to may be to manipulate stimuli that precede behaviour (antecedents). This means that one has to rely on that the stimuli in the room (including experimenter behaviour) will elicit the responses one wishes to see, based on the child's existing preferences and reinforcement history. Children who can understand verbal rules may be able to modify their behaviour directly, without repetition because the rule specifies both the target behaviour and the reward. However, for low functioning children who do not understand verbal rules, repetition of the target behaviour and discriminative delivery of concrete rewards is necessary to achieve positive reinforcement. It might mean that the first trials of the experiment have to be rejected and considered as practise trials.

When using social and verbal rewarding like praising the child, it should be kept very short and clear (e.g. good job!) and it is good to consider the intervals when to use it. It has, indeed, been found that young children with ASD benefited from the social, verbal reward which was a systematic, clear praise and appeared almost simultaneously with the child's response (Jones et al. 2013). It might, however, turn out that the purely verbal reward is not enough and the concrete, visual reward, which is delivered immediately after the desired behaviour, is needed. Tokens, stickers and snacks have been used as a concrete reward (cf., Kylliäinen et al. 2006; de Wit et al. 2008). When using edible rewards (e.g. raisins, candies or cookies), it is good to keep in mind that the size of the reward is small enough that it would not take too long to eat it as the jaw movements give artefacts to most of the measurements, especially to EEG and MEG measurements. The type of reward is one of those things that has to be confirmed with the parents before the experiment; children with ASD are quite often on a special diet. The interval of reinforcing depends of the task and the age of the child. It should be, however, as systematic as possible for all participating children.

If the experiment can be divided into parts, a reward can be delivered after each part is finished. Some children may be motivated by the use of explicit rewards systems that specify future rewards and their associated target behaviours (e.g. keep quiet and sit still). Such systems can help the child anticipate the time that is left of the experiment. For example, 
the child could be shown a card with five empty slots representing the length of the task and the reward sticker would be attached to the empty slot right after the specific part is finished. Alternatively, the progress in experimental trials could be visualised on the computer screen (Kylliäinen et al. 2006). This strategy is not optimal with the very young children and more simple strategies could be better with them. The child could, for example, be shown a heart rate lead and a candy and be told: "first lead, then candy" by emphasising the words first and then. Of course, even this practice requires some basic capability for verbal rule comprehension and following. Picture cards can be used in a similar way (e.g. first [picture A showing target behaviour], then [picture B showing reward]; Fig. 2).

The methods for guiding the child during the experiment largely resample the intervention principles and other clinical methods used in children with ASD (i.e. visual structuring, anticipating, reinforcing). The use of these kinds of intervention strategies could be more familiar to some of the children than others depending on their experience of the type intervention. This issue is particularly important in using EEG to test effects of early intervention (cf., Dawson et al. 2012). One further issue to mention is that children with ASD and low developmental level find it usually difficult to choose. In case the experimenter is using choosing as a method for rewarding the child (e.g. letting the child to choose the colour of the candy, reward stickers, the colour of the lead), it is important to limit the number of which to choose (max. of two options) and when to choose.

\section{Experimenter Characteristics}

A clinically experienced experimenter who also knows the purpose of the study well enough is the right person to lead the child through the experiment. Anticipating and preventing the child's unwanted actions are the key issues to keep in mind when supporting the child with ASD. It is possible to anticipate the need of a break or extra reinforcement or even prevent child of tearing the leads off by closely observing the child.

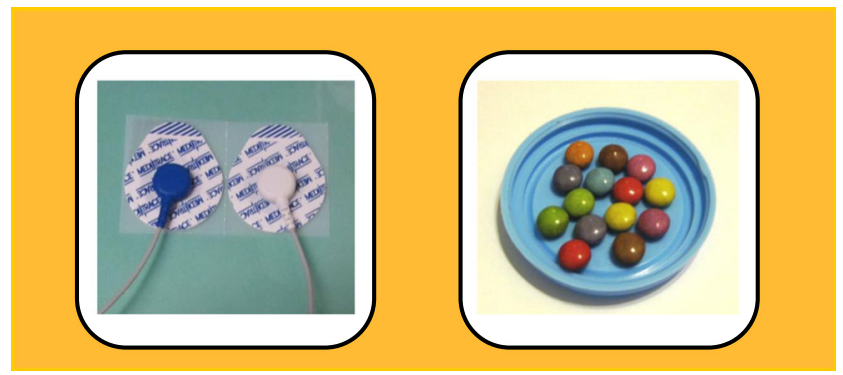

Fig. 2 An example of a card in which the target behaviour (on left) and the reward (on right) are illustrated
One of the most difficult tasks for the experimenter is to pace one's own actions. The experimenter has to be quick and calm at the same time. For example, the experimenter cannot show her/his own distress when the things are not going smoothly. In order to be quick and smooth in the preparations and other procedure tasks, the experimenter needs practice. The experimenter should be cautious not to talk too much. This is important not only to avoid the child with ASD to get anxious as he/she cannot necessarily understand what is said and does not benefit of the social chat, but also in order to keep experimenter's own behaviour relatively similar between the children with and without special needs. Instead of verbal instructions, the experimenter may sometimes prefer to use his/her own hands to support the child, for example, by placing one's own hand on top of the child's hand in order to prevent tapping on a table. These actions should be done if possible during the inter stimulus intervals. Especially, when measuring psychophysiological arousal which is affected by touch, it is important to avoid touching the child. Some children with ASD cannot bear gentle touch or touching at all. The experimenter needs to test which type of touch is tolerable during the preparation/warm up period.

During the experiment, it is sometimes optimal if the experimenter could position her/himself right behind the child. That way, the experimenter is not in the visual field of the child and will not bias the child's attention to any particular side. Sitting on the side (but still slightly behind the child) can have some advantages as well. First, it is easier to intervene if the child's behaviour can damage or interfere with the equipment (e.g. touching the screen). The popular Tobii T120 eye tracker systems have buttons below the screen, fully visible and within reach of the child that allow the child to, among other things, turn off the screen. Another advantage with being slightly on the side is that the experimenter can observe the child's face (and hands) better, which may be useful in some circumstances. Finally, it is possible that being visible in the periphery can cause some children to relax more, since they 'know where you are'. This may in turn potentially reduce the child's tendency to orient to the experimenter. In MEG measurements, it is often impossible to be behind the child due to shape of the scanner. This problem was solved in Kylliäinen et al.'s (2006) study by placing two supporting experimenters each side of the child. It was found, however, in the pilot measurements, that two experimenters in the magnetically shield room caused artefacts. Providing inflatable chairs for the experimenters resolved this problem.

The optimal place for a parent in the experiment room has to be considered too. In general the parent is good to be present as she/he can be helpful not only for the child but also for the experimenter in case there are difficult situations. The parent should not be placed in the visual field of the child. In the eye tracking studies parents have found it interesting to observe child's online eye movements from the controlling screen. 


\section{Difficult Situations}

Despite all the careful preparations, there might be difficult situations during the experimental sessions. Technical problems are very hard to completely avoid when utilising computers and psychophysiological measures. The experimenters have to plan the procedure what to do when the technical problem occurs. It has been found as a good strategy that when one experimenter is solving technical issues another experimenter is attracting the child's attention to something interesting. There could be an attractive toy available for the child to fiddle or there could be time for a short snack break if more time is needed to get ready to carry on the measurements. It has to be taken care of that the toy is not too attractive that the child does not want to give it away when the technical problem is solved and is time to carry on the experiment.

Another typical difficulty arises when the child's attention drops during the experiment. The children with ASD might, for example, habituate to the attention getters and become restless and fidgety. These problems could be anticipated by having another kind of the attention getter for different tasks or for another half of the experiment. If the child's attention to the stimulus drops during the experiment, most research questions would tolerate some kind of strategy to reorient the child's attention. If other more systematic methods of getting attention back to the task do not work, the experimenter may reorient the child by pointing or talking to the child (or parent). Doing so, one should carefully consider whether this procedure could introduce confounds. For example, manually pointing to the desired stimulus might have different influences on processing in different groups due to for example joint attention effects.

When studying young children with ASD, the most challenging situation during the experiment is when the child does not comply with task instructions or does not respond in the desired way to experimenter's prompts (e.g. leaves the room or refuses to wear a EEG-net). This will happen from time to time despite rigorous preparations. In these situations, the experimenter needs to evaluate whether one should abort the task, or whether it is worthwhile to give another try. Here, care should be taken not to put too much pressure on the child. The information from the parents can be valuable in this situation. Given their experience with the child, the parents may be in the best position to judge whether or not their child can continue with the experiment, and what is necessary to get cooperation of the child. It is crucial to keep positive towards the parents and normalise the situation reminding that the study demands are high and their child is not the only one who refuses some parts of the experiment. On the other hand, the parents might actually be more tolerant for their child's resisting than the experimenter. The experimenter cannot leave it completely up to the parents to judge, as they may feel under press to perform 'well' (with their child) in a research setting (attitudes towards research may differ a lot depending on cultural factors, which the experimenter should be aware of). In case the experiment has be to aborted, it is advisable to ask parents whether they think it could be possible to modify the procedure and/or try to carry on another time or after a break. Sometimes, it could be more ethical to carry on the experiment even though it seems highly likely that the data cannot be used (e.g. too many confounding factors have been introduced, there are too few trials or too much movement artefact). It is important not only for the parents to feel that their visit was useful but also to train the child to these types of situations. It might, for example, be helpful for the child and the parents to cope in the future medical examinations that are necessary to accomplish with the child.

\section{Data Handling}

The data analyses methods which are highly specific in varying techniques are naturally out of the scope of this article. There are, however, a couple of result-related issues to think through when studying special populations such as young children with ASD. At first, it should be considered how to give instant feedback for the child and the parents and how to communicate about the findings in ethical way.

\section{Feedback to the Child and the Parents}

It is quite common procedure and approved in many national ethical standards to give a small gift for the child at the end of the measuring visit. The reward at the end of the measurement can also be something that has been promised for the child during the experiment, e.g. that the child could watch the rest of the break video when finished or fiddle with some object that has had to be removed from the child during the measurement. Apart from rewarding the child, it is essential to give some instant feedback about the child's performance to the parents. This feedback is recommended, however, to be limited only to aspects about child's behaviour and coping in the experiment as the findings are not yet analysed and it is too early to conclude something about the child's performance in relation to the study question.

The parents are quite often very eager to hear about the individual findings of their child. It should be considered what kind of individualised information is ethical to provide to the parents not to increase the parental worry of the child with special needs or emphasise irrelevant aspects of the child's behaviour unnecessarily. The feedback of the research findings has to follow always the guidelines of the institutional ethical board. It is a controversial issue to give parents individual findings of their child together with the group findings. In case the parents interpret that their child's behaviour is not in line with the group findings in which the child belongs, it may unduly worry the parents. 
In eye tracking studies, short gaze replays have been shown to the parents after the experiment (cf., Falck-Ytter et al. 2013a, b, c). The experience from that study was that it was easy to communicate in a positive and informal way about individual data that was highly appreciated by all parents. In a MEG study (Kylliäinen et al. 2006), individual printouts of face specific activity and a photo of the child during the preparation phase was sent afterwards to the families together with a thank you letter of participation. Another possibility is to give the individual feedback from the clinical assessments or questionnaires which have been done as a part of the research procedure. It has also been found to be a useful strategy to give feedback of the study purpose and its findings in a seminar organised solely for the parents of the participating children (cf., Kylliäinen et al. 2006, 2012). In some countries (e.g. Sweden), participants have the legal right to get access to all the data associated with their child, once per year, upon request.

\section{Online Strategies for Recording Child's Behaviour and Other Factors}

It is a common procedure in experimental studies to have a $\log$ sheet for recording all the experiment relevant aspects of the particular measurement. These kinds of notes are especially crucial for controlling the data quality when studying young children with additional needs. The $\log$ sheet should include options to write down for example, responses to instructions, deviations from standard protocol, and other noteworthy events that occur during the experiment. These log sheets can also include methods specific issues, such as a recalibration for eye tracking or high impedances of some EEG channels. It is also important to collect all background information that might have affected the measurement, e.g. how the child has slept in the previous night or when the child has eaten. All the log sheet information is not only needed for rejecting the data but also for exploring afterwards whether the alterations and changes affected the results.

In addition, it is often essential to record a video of child's behaviour during the experiment. The recording should be able to be synchronised with the psychophysiological measures. In that way, all episodes where the child is talking, moving or not attending and looking can be excluded from the analyses. Depending on the study question sometimes even two video cameras are required. The offline video analysis is very time consuming and sometimes online coding of the visual attention in ERP paradigms has been used (cf., Webb et al. 2011; Dawson et al. 2012) together with recording all the exceptional situations during the measurement (e.g. the parent is talking with the experimenter).

\section{Offline Strategies to Reduce Data Loss}

No matter which measurements are being used, it is essential to maximise the data acquisition by minimising the attrition rate also in the phase of analyses. If enough attention is paid to the issues of preparation and supporting the child during the experiment, attrition rates in children with ASD can be quite similar to those in TD children. For instance, in Ruysschaert et al.'s (2014) study, $11.4 \%$ of children with ASD and $4.7 \%$ of typically developing children refused to start or complete the experiment. In addition to that type of dropout, the attrition rate could get even higher at the analyses phase. In Ruysschaert et al.'s (2014) study, $41.9 \%$ of the children with ASD and $47.5 \%$ of the control children were excluded from the final sample because of insufficient artefact-free data.

Given that the measurement of young children with ASD is demanding and time-consuming with all the clinical measurements for qualifying the diagnosis and preparations for helping the child to cope the experimental setting, it is not preferable to reject an entire participant due to insufficient amount of good data. Basically, this means that it is necessary to invest more time doing data cleaning than it would needed for a study in which solely typically developing children are being investigated. This can include specifying data inclusion/exclusion criteria differently than you would for a typical sample. For example, depending on the study question one could end up tolerating some behaviour that would not be tolerable in the studies with typically developing children (e.g. minor fidgeting movement of the feet). Sometimes one needs to analyse even part of the trial in order to reduce the number of excluded trials or even participants.

Often, one ends up with more artifactual data in the ASD group and the developmental delay group than in the group of typically developing children. Care should be taken that in the phase of data cleaning, the data exclusion and inclusion criteria have to be same for all the participating groups of children. One possibility is to include the same amount of trials in the typically developing children than in the groups of children with additional needs although there would have been more artefact-free data in the former group. It should be made sure, however, that the included trials are from the same part of the measurement time in all of the groups. It has been shown in EEG study that typically developing infants had varying ERP-findings depending whether the first half or last half of the trials was analysed (Stets et al. 2012).

\section{Discussion}

In this article, we aimed to make explicit the practical strategies and other specific issues to be considered when conducting psychophysiological experiments in young children with ASD and other neurodevelopmental conditions. We 
wanted to document and increase awareness of these relatively informal strategies in order to maximise data acquisition and quality and to facilitate cross-study comparisons. We focused on the range of common, non-invasive psychophysiological measures such as EEG, MEG, eye tracking and measurements of heart rate and skin conductance. The specific features of (a) design, (b) preparing for the measurement visit, (c) conducting the experiment and (d) the data handling were discussed. Due to great variability in the participating children, used methods and study questions, it was not desirable to try to create firm recommendations. We have summarised the covered issues as so called Experimenter's check list (Table 2) of issues that a research team needs to think through from the practical point of view when planning the psychophysiological study in young children with ASD. It should be emphasised, however, that not all of the issues are autism specific but apply to most young children and to children with additional needs. The experimental studies in young, low functioning children with ASD quite often necessitates a control group of children who have developmental delay without autistic behaviour in order to confirm

Table 2 Summary of key issues that should be considered when studying young children with ASD in psychophysiological experiments

Experiment's check list

- Is the overall research design realistic given the population characteristics?

- Have possible sensory abnormalities been taken into account in the stimuli selection?

- Is the measurement time long enough to get enough usable data and at the same time short enough that a child with special needs can cope it?

- Are the stimuli sufficiently attention grabbing and motivating?

- Can one use an active task to increase attention and motivation?

- Is the testing environment optimal for the purpose (e.g. few distractors)?

- How can parent's knowledge of their child be used to individualize procedures?

- How should the child be familiarized to the people and environment upon arrival? Is desensitising necessary?

- What is the optimal seating arrangement (child, experimenter and parent) during the experiment?

- Is the experimenter clinically experienced and how could she/he help the child during the experiment?

- Which behavioural control strategies will be used?

-What is the plan for unexpected difficulties (e.g. technical problems, child behaviour) during the measurement?

- Is the experiment log sheet adequate for efficient recording of unexpected events?

- Is audio-visual recording equipment optimally chosen and placed for the purpose of the study (e.g. does the video camera capture the face of the child?)?

- What kind of feedback can be given in order to reward parents and children for their time/effort and motivate them for future research participation?

- How do the issues arising during data collection influence the subsequent (pre-)processing of data? that the findings are specific for autism and not only for general developmental delay (cf., Charman 2004). Thus, these practical principles are thought to be useful also in measuring the control group that is matched according to the developmental age.

Throughout the introduced strategies and guidelines the one key topic has been the difficulty in balancing between how much individualisation of the procedure is tolerable in order to get data, and how does that lack of standardisation influence the results in different groups of children. Some degree of adaptation can be essential for children with ASD, but if things are adapted too much, then it might prevent the comparisons with the control groups. For example, in case one has to reorient a child to the screen every trial or only just once at the start of the experiment, it has to be considered how that influences task performance.

Any researcher involved in studies with young children with ASD and other neurodevelopmental conditions will need to somehow achieve a balance between standardization and individualisation. While a high degree of standardisation of test protocols and procedures is intuitively the best option, it may be unrealistic when studying this population. There is of course no way to describe a general rule for this balancing act. One has to consider the research question and carefully consider what aspects of the study need to be standardised in order to be able to draw conclusions about that particular question. For measures that are not sensitive to contextual or procedural factors, a large degree of individualisation is acceptable. For psychological measures, however, it is often not known to what degree they are influenced by contextual and procedural factors, leaving much up to the researcher's judgement. To guide this process, it may be useful to think of psychological functions as part of a hierarchy. If the psychological function of interest is high up in this hierarchy (e.g. 'mentalising'), one should standardise the test of this function. However, it may be necessary to individualise procedures to ensure that psychological functions further down in the hierarchy (e.g. attention, motivation) are balanced between groups during the experiment. If groups are not matched at these basic levels, it will be difficult to draw conclusions about the any group differences at the higher level. In general, if one cannot match on a certain variable (i.e. achieving control by keeping something constant), one should at least try to measure it and explore its role statistically.

These practical guidelines are not only essential for maximising the quality and acquisition of the data but also for doing ethical research in the special population of children. The clinical knowledge about the specific strategies how to handle young children with ASD is required in a research team. The clinical experience is also needed from the perspective of working sensitively with parents, who could generally be much more sensitive to their child's performance than parents of typically developing children. This can be especially important with the youngest children because they have fairly new diagnoses and so families are adjusting to their new status. 
Taken together, the range of practical strategies and issues to consider is quite wide and measuring an individual child could be rather challenging when conducting psychophysiological studies in young children with ASD and other neurodevelopmental conditions. Researchers have to find the right balance between individualisation and standardisation in order to maximise data quantity and quality. Despite all the challenges in this field, studying young, possibly lowfunctioning children with ASD is essential goal in autism research. Hopefully, by making these practical strategies and specific issues to consider as explicit as possible and giving some basic guidelines to follow, it is possible to accelerate the pace of the research in this important area.

Acknowledgments This work was supported by the ESF COST Action BM1004 Enhancing the Scientific Study of Early Autism (ESSEA), and the Innovative Medicines Initiative Joint Undertaking under grant agreement $\mathrm{n}^{\circ} 115300$, resources of which are composed of financial contribution from the European Union's Seventh Framework Programme (FP7/ 2007-2013) and EFPIA companies' in kind contribution. The work of A.K was supported by the Academy of Finland (project \#250480). This work of T.F-Y was supported by the Swedish Research Council in partnership with FAS, FORMAS, and VINNOVA (Cross-disciplinary research program concerning children's and young people's mental health; grant number 259-2012-24) and The Bank of Sweden Tercentenary Foundation (P12-0270:1).

\section{Conflict of Interest No conflict of interest}

Open Access This article is distributed under the terms of the Creative Commons Attribution License which permits any use, distribution, and reproduction in any medium, provided the original author(s) and the source are credited.

\section{References}

American Psychiatric Association. (2013). The diagnostic and statistical manual of mental disorders: DSM 5 bookpoint US.

Ashwin, E., Ashwin, C., Rhydderch, D., Howells, J., \& BaronCohen, S. (2009). Eagle-eyed visual acuity: An experimental investigation of enhanced perception in autism. Biological Psychiatry, 65(1), 17-21.

Baker, A. E., Lane, A., Angley, M. T., \& Young, R. L. (2008). The relationship between sensory processing patterns and behavioural responsiveness in autistic disorder: a pilot study. Journal of Autism and Developmental Disorders, 38(5), 867-875.

Barr, R., Muentener, P., Garcia, A., Fujimoto, M., \& Chávez, V. (2007). The effect of repetition on imitation from television during infancy. Developmental Psychobiology, 49(2), 196-207.

Ben-Sasson, A., Hen, L., Fluss, R., Cermak, S. A., Engel-Yeger, B., \& Gal, E. (2009). A meta-analysis of sensory modulation symptoms in individuals with autism spectrum disorders. Journal of Autism and Developmental Disorders, 39(1), 1-11.

Blakemore, S., Tavassoli, T., Calò, S., Thomas, R. M., Catmur, C., Frith, U., \& Haggard, P. (2006). Tactile sensitivity in Asperger syndrome. Brain and Cognition, 61(1), 5-13.
Carver, L. J., Meltzoff, A. N., \& Dawson, G. (2006). Event-related potential (ERP) indices of infants' recognition of familiar and unfamiliar objects in two and three dimensions. Developmental Science, 9(1), 51-62.

Charman, T. (2004). Matching preschool children with autism spectrum disorders and comparison children for language ability: methodological challenges. Journal of Autism and Developmental Disorders, 34(1), 59-64.

Chawarska, K., \& Shic, F. (2009). Looking but not seeing: atypical visual scanning and recognition of faces in 2 and 4-year-old children with autism spectrum disorder. Journal of Autism and Developmental Disorders, 39(12), 1663-1672.

Cléry, H., Bonnet-Brilhault, F., Lenoir, P., Barthelemy, C., Bruneau, N., \& Gomot, M. (2013). Atypical visual change processing in children with autism: an electrophysiological study. Psychophysiology, $50(3), 240-252$.

Dawson, G., Jones, E. J., Merkle, K., Venema, K., Lowy, R., Faja, S., \& Winter, J. (2012). Early behavioral intervention is associated with normalized brain activity in young children with autism. Journal of the American Academy of Child \& Adolescent Psychiatry, 51(11), $1150-1159$.

de Wit, T. C., Falck-Ytter, T., \& von Hofsten, C. (2008). Young children with autism spectrum disorder look differently at positive versus negative emotional faces. Research in Autism Spectrum Disorders, 2(4), 651-659.

Dyckens, E. M., \& Lense, M. (2011). Intellectual disabilities and autism spectrum disorder: A cautionary note. In D. G. Amaral, D. H. Geschwind, \& G. Dawson (Eds.), Autism spectrum disorders (pp. 263-269). New York: Oxford University Press.

Falck-Ytter, T. (2010). Young children with autism spectrum disorder use predictive eye movements in action observation. Biology Letters, 6(3), 375-378.

Falck-Ytter, T., Fernell, E., Hedvall, A. L., von Hofsten, C., \& Gillberg, C. (2012). Gaze performance in children with autism spectrum disorder when observing communicative actions. Journal of Autism and Developmental Disorders, 42(10), 2236-2245.

Falck-Ytter, T., Rehnberg, E., \& Bölte, S. (2013a). Lack of visual orienting to biological motion and audiovisual synchrony in 3year-olds with autism. PloS One, 8(7), e68816.

Falck-Ytter, T., von Hofsten, C., Gillberg, C., \& Fernell, E. (2013b). Visualization and analysis of eye movement data from children with typical and atypical development. Journal of Autism and Developmental Disorders, 43(10), 2249-2258.

Falck-Ytter, T., Bolte, S., \& Gredeback, G. (2013c). Eye tracking in early autism research. Journal of Neurodevelopmental Disorders, 5(1), 28. doi:10.1186/1866-1955-5-28.

Gepner, B., \& Féron, F. (2009). Autism: a world changing too fast for a mis-wired brain? Neuroscience \& Biobehavioral Reviews, 33(8), $1227-1242$.

Gerrard, S., \& Rugg, G. (2009). Sensory impairments and autism: a reexamination of causal modelling. Journal of Autism and Developmental Disorders, 39(10), 1449-1463.

Gomot, M., Blanc, R., Clery, H., Roux, S., Barthelemy, C., \& Bruneau, N (2011). Candidate electrophysiological endophenotypes of hyperreactivity to change in autism. Journal of Autism and Developmental Disorders, 41(6), 705-714.

Grice, S. J., Halit, H., Farroni, T., Baron-Cohen, S., Bolton, P., \& Johnson, M. H. (2005). Neural correlates of eye-gaze detection in young children with autism. Cortex, 41(3), 342-353.

Hallmayer, J., Cleveland, S., Torres, A., et al. (2011). Genetic heritability and shared environmental factors among twin pairs with autism. Archives of General Psychiatry, 68(11), 1095-1102. 
Hirstein, W., Iversen, P., \& Ramachandran, V. S. (2001). Autonomic responses of autistic children to people and objects. Proceedings. Biological Sciences the Royal Society, 268(1479), $1883-1888$.

Holmqvist, K., Nyström, M., Andersson, R., Dewhurst, R., Jarodzka, H., \& Van de Weijer, J. (2011). Eye tracking: a comprehensive guide to methods and measures. Oxford University Press.

Itier, R. J., \& Batty, M. (2009). Neural bases of eye and gaze processing: the core of social cognition. Neuroscience \& Biobehavioral Reviews, 33(6), 843-863.

Jones, W., Carr, K., \& Klin, A. (2008). Absence of preferential looking to the eyes of approaching adults predicts level of social disability in 2year-old toddlers with autism spectrum disorder. Archives of General Psychiatry, 65(8), 946-954.

Jones, E., Webb, S., Estes, A., \& Dawson, G. (2013). Rule learning in autism: the role of reward type and social context. Developmental Neuropsychology, 38(1), 58-77.

Jones, E. J., Gliga, T., Bedford, R., Charman, T., \& Johnson, M. H. (2014). Developmental pathways to autism: a review of prospective studies of infants at risk. Neuroscience \& Biobehavioral Reviews, 39, 1-33.

Joseph, R. M., Ehrman, K., McNally, R., \& Keehn, B. (2008). Affective response to eye contact and face recognition ability in children with ASD. Journal of the International Neuropsychological Society, 14(06), 947-955.

Khalfa, S., Bruneau, N., Rogé, B., Georgieff, N., Veuillet, E., Adrien, J., \& Collet, L. (2004). Increased perception of loudness in autism. Hearing Research, 198(1), 87-92.

Klin, A., Lin, D. J., Gorrindo, P., Ramsay, G., \& Jones, W. (2009). Twoyear-olds with autism orient to non-social contingencies rather than biological motion. Nature, 459(7244), 257-261.

Kylliäinen, A., \& Hietanen, J. K. (2006). Skin conductance responses to another person's gaze in children with autism. Journal of Autism and Developmental Disorders, 36(4), 517-525.

Kylliäinen, A., Braeutigam, S., Hietanen, J. K., Swithenby, S. J., \& Bailey, A. J. (2006). Face- and gaze-sensitive neural responses in children with autism: a magnetoencephalographic study. The European Journal of Neuroscience, 24(9), 2679-2690.

Kylliäinen, A., Wallace, S., Coutanche, M. N., Leppänen, J. M., Cusack, J., Bailey, A. J., \& Hietanen, J. K. (2012). Affective-motivational brain responses to direct gaze in children with autism spectrum disorder. Journal of Child Psychology and Psychiatry, 53(7), 790797.

Leekam, S. R., Nieto, C., Libby, S. J., Wing, L., \& Gould, J. (2007). Describing the sensory abnormalities of children and adults with autism. Journal of Autism and Developmental Disorders, 37(5), 894-910.

Lloyd-Fox, S., Blasi, A., \& Elwell, C. (2010). Illuminating the developing brain: the past, present and future of functional near infrared spectroscopy. Neuroscience \& Biobehavioral Reviews, 34(3), 269284.

Lord, C., Rutter, M., DiLavore, P., Risi, S., Gotham, K., \& Bishop, S. (2012). Autism diagnostic observation schedule: ADOS-2 Western Psychological Services Torrance.

Marco, E. J., Hinkley, L. B., Hill, S. S., \& Nagarajan, S. S. (2011). Sensory processing in autism: a review of neurophysiologic findings. Pediatric Research, 69, 48R-54R.

Orekhova, E. V., Stroganova, T. A., Prokofyev, A. O., Nygren, G., Gillberg, C., \& Elam, M. (2008). Sensory gating in young children with autism: relation to age, IQ, and EEG gamma oscillations. Neuroscience Letters, 434(2), 218-223.

Patriquin, M. A., Scarpa, A., Friedman, B. H., \& Porges, S. W. (2013). Respiratory sinus arrhythmia: a marker for positive social functioning and receptive language skills in children with autism spectrum disorders. Developmental Psychobiology, 55(2), 101112.

Peltola, M. J., Leppänen, J. M., Palokangas, T., \& Hietanen, J. K. (2008). Fearful faces modulate looking duration and attention disengagement in 7-month-old infants. Developmental Science, 11(1), 60-68.

Reynolds, S., \& Lane, S. J. (2008). Diagnostic validity of sensory overresponsivity: a review of the literature and case reports. Journal of Autism and Developmental Disorders, 38(3), 516-529.

Ruysschaert, L., Warreyn, P., Wiersema, J. R., Metin, B., \& Roeyers, H. (2013). Neural mirroring during the observation of live and video actions in infants. Clinical Neurophysiology, 124(9), $1765-1770$.

Ruysschaert, L., Warreyn, P., Wiersema, J. R., Oostra, A., \& Roeyers, H. (2014). Exploring the role of neural mirroring in children with autism spectrum disorder. Autism Research. doi:10.1002/aur.1339.

Sandin, S., Lichtenstein, P., Kuja-Halkola, R., Larsson, H., Hultman, C. M., \& Reichenberg, A. (2014). The familial risk of autism. JAMA, 311(17), 1770-1777.

Schoen, S. A., Miller, L. J., Brett-Green, B., \& Hepburn, S. L. (2008). Psychophysiology of children with autism spectrum disorder. Research in Autism Spectrum Disorders, 2(3), 417-429.

Senju, A., \& Csibra, G. (2008). Gaze following in human infants depends on communicative signals. Current Biology, 18(9), 668-671.

Senju, A., Tojo, Y., Yaguchi, K., \& Hasegawa, T. (2005). Deviant gaze processing in children with autism: an ERP study. Neuropsychologia, 43(9), 1297-1306.

Shimada, S., \& Hiraki, K. (2006). Infant's brain responses to live and televised action. NeuroImage, 32(2), 930-939.

Sigman, M., Dissanayake, C., Corona, R., \& Espinosa, M. (2003). Social and cardiac responses of young children with autism. Autism : The International Journal of Research and Practice, $7(2), 205-216$.

Snyder, K., Webb, S. J., \& Nelson, C. A. (2002). Theoretical and methodological implications of variability in infant brain response during a recognition memory paradigm. Infant Behavior and Development, 25(4), 466-494.

Stets, M., Stahl, D., \& Reid, V. M. (2012). A meta-analysis investigating factors underlying attrition rates in infant ERP studies. Developmental Neuropsychology, 37(3), 226-252.

Stiegler, L. N., \& Davis, R. (2010). Understanding sound sensitivity in individuals with autism spectrum disorders. Focus on Autism and Other Developmental Disabilities, 25(2), 67-75.

Swanson, M. R., \& Siller, M. (2013). Patterns of gaze behavior during an eye-tracking measure of joint attention in typically developing children and children with autism spectrum disorder. Research in Autism Spectrum Disorders, 7(9), 1087-1096.

Tomchek, S. D., \& Dunn, W. (2007). Sensory processing in children with and without autism: a comparative study using the short sensory profile. The American Journal of Occupational Therapy : Official Publication of the American Occupational Therapy Association, 61(2), 190-200.

Warreyn, P., Ruysschaert, L., Wiersema, J. R., Handl, A., Pattyn, G., \& Roeyers, H. (2013). Infants' mu suppression during the observation of real and mimicked goal-directed actions. Developmental Science, 16(2), 173-185.

Watson, L. R., Roberts, J. E., Baranek, G. T., Mandulak, K. C., \& Dalton, J. C. (2012). Behavioral and physiological responses to childdirected speech of children with autism spectrum disorders or typical development. Journal of Autism and Developmental Disorders, 42(8), 1616-1629.

Webb, S. J., Jones, E. J., Merkle, K., Namkung, J., Toth, K., Greenson, J., \& Dawson, G. (2010). Toddlers with elevated autism symptoms 
show slowed habituation to faces. Child Neuropsychology, 16(3), 255-278.

Webb, S. J., Jones, E. J., Merkle, K., Venema, K., Greenson, J., Murias, M., \& Dawson, G. (2011). Developmental change in the ERP responses to familiar faces in toddlers with autism spectrum disorders versus typical development. Child Development, 82(6), 1868-1886.

Webb, S. J., Bernier, R., Henderson, H. A., Johnson, M. H., Jones, E. J., Lerner, M. D., Townsend, J. (2013). Guidelines and best practices for electrophysiological data collection, analysis and reporting in autism. Journal of Autism and Developmental Disorders, 1-19. doi: 10.1007/s10803-013-1916-6

\section{Highlights}

- Practical strategies of psychophysiological studies in young children with ASD are introduced.

- The focus is on technologies such as EEG, MEG, eye tracking and measures of physiological arousal.

- A key theme is the difficulty in balancing between the procedural adaptations and standardisation.

- These practical strategies are expected to ease and accelerate research in this important field. 